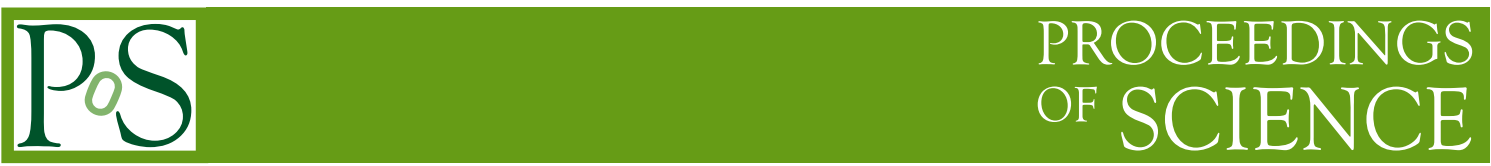

\title{
Sterile Neutrino Searches with MINOS/MINOS+
}

\author{
Leigh H. Whitehead ${ }^{* \neq \ddagger}$ \\ Department of Physics and Astronomy, University College London, Gower Street, London, \\ WC1E 6BT, United Kingdom \\ E-mail: leigh.howard.whiteheadecern.ch
}

\begin{abstract}
The MINOS and MINOS+ experiment ran from 2005 until 2016 with two detectors at baselines of $1 \mathrm{~km}$ and $735 \mathrm{~km}$ exposed by the NuMI beam from Fermilab. The NuMI beam neutrino flux peaked at an energy of $3 \mathrm{GeV}$ during the MINOS era in both $v_{\mu}$ and $\bar{v}_{\mu}$ modes. The MINOS+ data were taken entirely in $v_{\mu}$ mode with a peak energy of $7 \mathrm{GeV}$. A study of three-flavour oscillations with the full MINOS sample and the first two years of data from MINOS+ measured $\Delta m_{32}^{2}=$ $(2.42 \pm 0.09) \times 10^{-3} \mathrm{eV}^{2}$ assuming the Normal Hierarchy, and $\Delta m_{32}^{2}=-\left(2.48_{-0.11}^{+0.09}\right) \times 10^{-3} \mathrm{eV}^{2}$ in the Inverted Hierarchy. Searches for both sterile neutrinos and sterile antineutrinos were performed with no significant evidence of a signal, hence exclusion limits were placed over six orders of magnitude of the sterile neutrino mass-splitting $\Delta m_{41}^{2}$. A joint analysis of the MINOS $v_{\mu}$ disappearance sample and the Daya Bay and Bugey- $3 \bar{v}_{e}$ disappearance samples excludes the LSND and MiniBooNE allowed regions for $\Delta m_{41}^{2}<0.8 \mathrm{eV}^{2}$ at $95 \%$ C.L.. Finally, an analysis searching for anomalous $v_{e}$ appearance above $6 \mathrm{GeV}$ in MINOS+ was used to set a limit on the allowed sterile neutrino parameter space.
\end{abstract}

38th International Conference on High Energy Physics

3-10 August 2016

Chicago, USA

\footnotetext{
* Speaker.

${ }^{\dagger}$ For the MINOS/MINOS+ Collaboration.

${ }^{\ddagger}$ Now at CERN, Geneva, Switzerland.
} 


\section{Introduction}

The MINOS/MINOS+ experiment [1] consists of two functionally equivalent magnetised tracking calorimeters formed from alternating layers of scintillator and steel. The two detectors are at baselines of $1 \mathrm{~km}$ and $735 \mathrm{~km}$ from the target that produces the NuMI beam [2] at Fermilab. MINOS ran from 2005 until 2012 in the low-energy (LE) beam configuration of the NuMI beam, with a peak energy of $3 \mathrm{GeV}$. The NuMI beam ran mostly in the $v_{\mu}$-dominated mode whereby positively charged mesons emergent from the target were focused using two magnetic horns to produce a $v_{\mu}$ beam. The polarity of the magnetic horns could also be reversed to generate a $\bar{v}_{\mu}$ enhanced beam with a significantly larger $\bar{v}_{\mu}$ component. The total LE exposure for MINOS was $10.56 \times 10^{20}$ protons-on-target (POT) in $v_{\mu}$-dominated mode and $3.36 \times 10^{20} \mathrm{POT}$ in $\bar{v}_{\mu}$-enhanced mode. The experiment was designed to measure the atmospheric scale oscillations, governed by the parameters $\theta_{23}, \theta_{13}$ and $\Delta m_{32}^{2}$.

In 2013, the experiment became MINOS+ and ran in the upgraded NuMI beam in mediumenergy (ME) mode until June 2016, with a peak energy of $7 \mathrm{GeV}$. The results presented here consider exposures of $2.99 \times 10^{20}$ POT and $5.80 \times 10^{20}$ POT in $v_{\mu}$-dominated mode corresponding to one and two years of MINOS+ data, respectively. The ME configuration for MINOS+ motivates searches beyond the standard model, since many non-standard effects become most evident in the high energy tail of the neutrino spectrum, away from the main three-flavour oscillation peak.

In particular, the $3+1$ sterile neutrino model will be considered. This is the minimal extension to include a fourth neutrino state, which must be sterile based on the results from LEP [3] showing there are only three neutrino species that couple to the $\mathrm{Z}$ boson with $m_{V}<0.5 m_{Z}$. This extension adds three new mixing angles $\left(\theta_{14}, \theta_{24}\right.$ and $\left.\theta_{34}\right)$ as part of an expanded $4 \times 4$ mixing matrix and and three additional mass-splittings, of which one, $\Delta m_{41}^{2}$, is independent. MINOS/MINOS+ has sensitivity to $\theta_{24}$ through searching for disappearance of both charged-current (CC) $v_{\mu}$ and neutralcurrent (NC) $v$ interactions, and small sensitivity to $\theta_{34}$ from the NC sample. The CC $v_{e}$ appearance analysis has sensitivity to a combination of $\theta_{14}$ and $\theta_{24}$.

\section{Standard Oscillations}

An update to the final MINOS three-flavour oscillations result [4] was performed using an additional $5.80 \times 10^{20}$ POT from the first two years of MINOS+ exposure and an additional year of atmospheric neutrino data. The event selection uses a k-Nearest-Neighbour method to preferentially select the CC $v_{\mu}$ and $\bar{v}_{\mu}$ interactions from a background of NC interactions from all neutrino flavours. The spectrum of CC $v_{\mu}$ and $\bar{v}_{\mu}$ events selected from the MINOS and MINOS+ beam is shown in Fig. 1 compared to the null oscillations case and the best-fit simulation. The filled histograms show the relative contributions to the spectrum from the MINOS and MINOS+ run periods, illustrating the difference between the LE and ME tunes of the NuMI beam.

The $90 \%$ C.L. allowed region of the atmospheric oscillation parameters $\theta_{23}$ and $\Delta m_{32}^{2}$ as a result of the fit is shown in Fig. 2. It is compared to the latest results from the NOvA [5] and T2K [6] experiments. The limits in the individual parameters can also be extracted:

$$
\Delta m_{32}^{2}= \begin{cases}(2.42 \pm 0.09) \times 10^{-3} \mathrm{eV}^{2} & \text { Normal Hierarchy } \\ -\left(2.48_{-0.11}^{+0.09}\right) \times 10^{-3} \mathrm{eV}^{2} & \text { Inverted Hierarchy }\end{cases}
$$




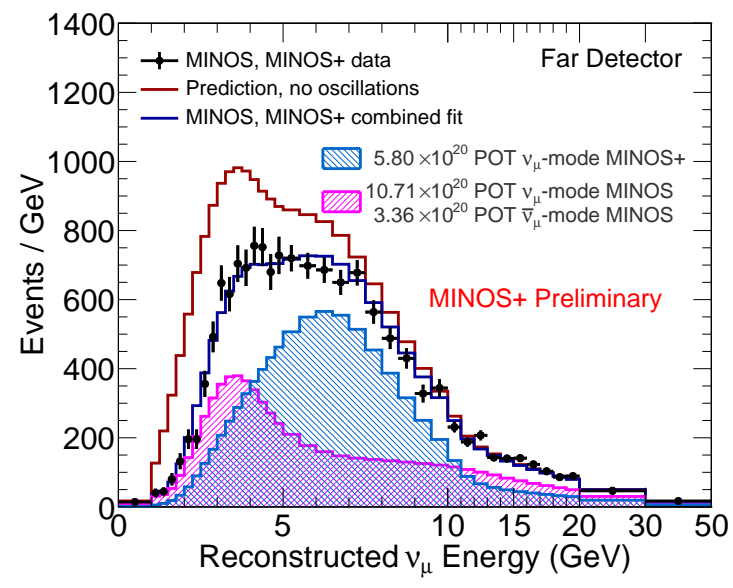

Figure 1: The observed CC $v_{\mu}$ and $\bar{v}_{\mu}$ spectrum for MINOS and MINOS+ (black points) compared to the no oscillations (red) and best-fit (blue) sim- Figure 2: The 90\% C.L. allowed region for the atulation. Also shown are the individual MINOS mospheric oscillation parameters measured by MI(pink) and MINOS+ (blue) contributions to the best- NOS and MINOS+ compared to the recent results fit simulation.

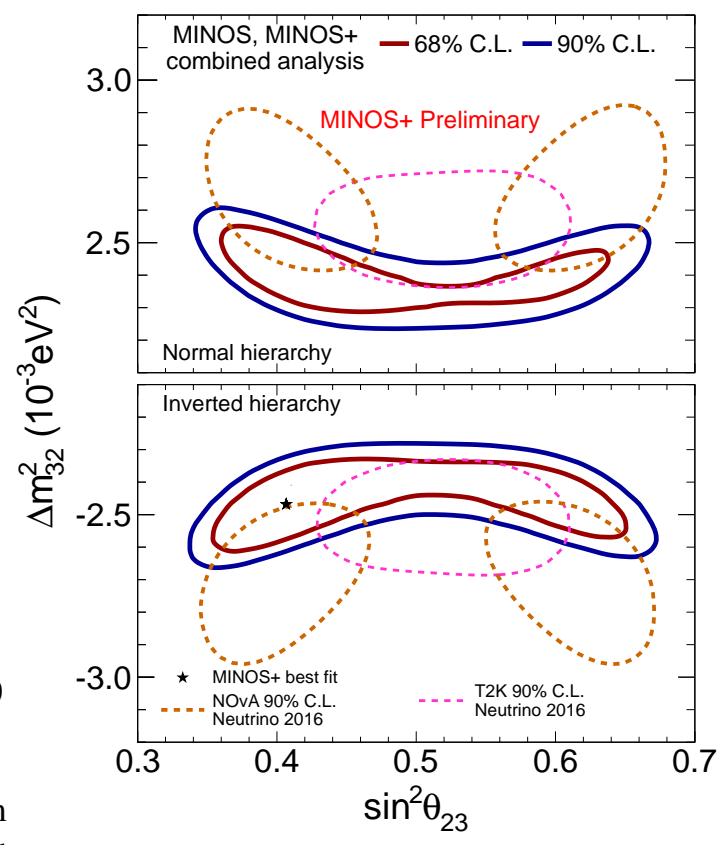

from the NOvA [5] and T2K [6] experiments.

$$
\sin ^{2} \theta_{23}= \begin{cases}0.35-0.65(90 \% \text { C.L. }) & \text { Normal Hierarchy } \\ 0.35-0.66(90 \% \text { C.L. }) & \text { Inverted Hierarchy }\end{cases}
$$

\section{Sterile Neutrinos}

The MINOS collaboration recently published a sterile neutrino analysis based on the full LE MINOS exposure [7]. A sterile neutrino could cause oscillations at short baselines meaning that the standard assumption of no oscillations upstream of the Near Detector is no longer valid, and hence the extrapolation procedure used in the standard oscillations analysis cannot be used. In order to deal with potential oscillations in both detectors and maximise the range of $\Delta m_{41}^{2}$ that could be probed, the analysis considered the Far-over-Near ratio of event spectra. This analysis used both $\mathrm{CC} v_{\mu}$ disappearance and $\mathrm{NC} v$ disappearance channels to search for a fourth neutrino state. The first two years of data from MINOS+ have also been analysed following the method developed for the MINOS analysis, and no significant evidence of a sterile neutrino was found. Figure 3 hence shows the $90 \%$ and $95 \%$ C.L. excluded regions in terms of $\Delta m_{41}^{2}$ and $\sin ^{2} \theta_{24}$ compared to a series of other experimental results, providing a strong exclusion over many orders of magnitude of $\Delta m_{41}^{2}$. 


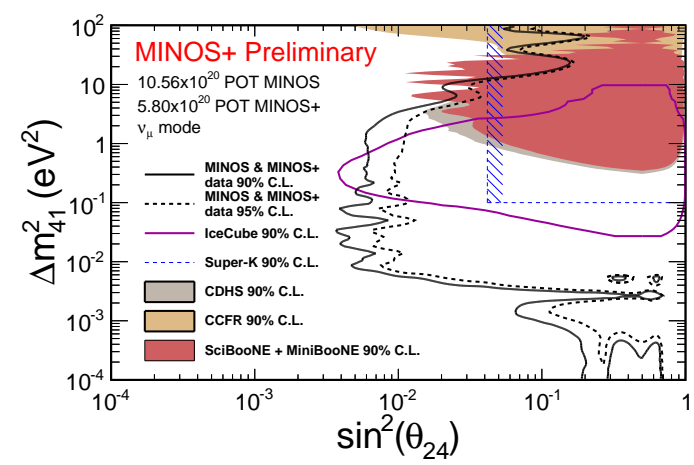

Figure 3: The $90 \%$ and $95 \%$ C.L. contours from the combined MINOS/MINOS+ sterile neutrino search compared to the recent result from IceCube [8], and further results from Super-K [9], CDHS [10], CCFR [11], and SciBooNE + MiniBooNE[12].

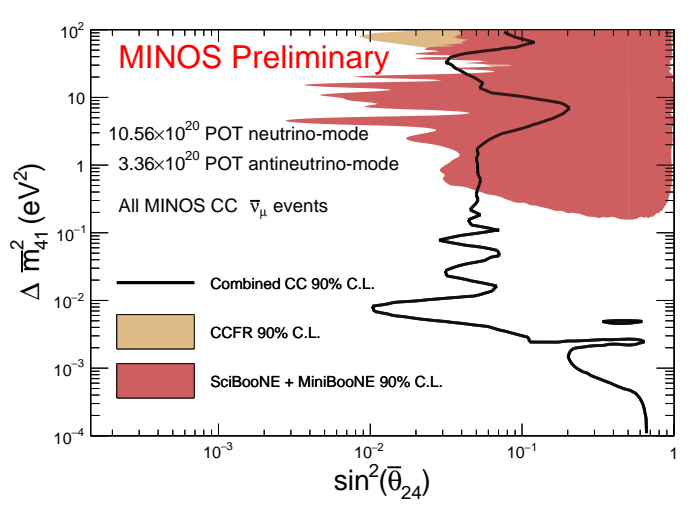

Figure 4: The $90 \%$ C.L. contour from the MINOS sterile antineutrino analysis compared results from CCFR [13] and SciBooNE + MiniBooNE [14].

\section{Sterile Antineutrinos}

The MINOS/MINOS+ detectors have the unique ability amongst long-baseline neutrino oscillation experiments to be able to select the charge of the muon outgoing from the $\mathrm{CC}$ interaction. This allows for the selection of a CC $\bar{v}_{\mu}$ sample to search for a sterile antineutrino. The sample of CC $\bar{v}_{\mu}$ interactions comprises two individual components, one from the $\bar{v}_{\mu}$-enhanced beam and the other from the $v_{\mu}$-dominated beam. The small $\bar{v}_{\mu}$ component in the $v_{\mu}$-dominated beam has higher energy because the antineutrinos come from the decay of parent mesons that are highly boosted along the axis of the magnetic horn. This makes the two samples complimentary as they probe different regions of the energy spectrum. The analysis was performed in the same way as the neutrino-mode analysis [7] and no sterile antineutrino signal was observed. Figure 4 shows the 90\% exclusion contour compared to results from CCFR [13] and SciBooNE + MiniBooNE [14].

\section{Combination with Daya-Bay and Bugey-3}

The MINOS $v_{\mu}$ disappearance sterile neutrino result [7] was combined with the $\bar{v}_{e}$ disappearance results from Daya Bay [15] and Bugey-3 [16] in a joint analysis [17] with the Daya Bay collaboration. Disappearance of $v_{\mu}$ in MINOS has sensitivity to $\theta_{24}$ and $\bar{v}_{e}$ disappearance in Daya Bay has sensitivity to $\theta_{14}$. The mass-squared splitting $\Delta m_{41}^{2}$ is common between the two channels, meaning that the combination of results, performed using the CLs technique, can probe $\sin ^{2} 2 \theta_{\mu e}=\sin ^{2} 2 \theta_{14} \sin ^{2} \theta_{24}$. The effective mixing angle $\sin ^{2} 2 \theta_{\mu e}$ is the parameter measured by LSND [18] and MiniBooNE [19]. Figure 5 shows the 90\% C.L. exclusion contour from MINOS and Daya Bay / Bugey-3 compared to allowed and excluded regions from other experiments, showing a very strong exclusion is set on the existence of the sterile neutrino for $\Delta m_{41}^{2}<0.8 \mathrm{eV}^{2}$. 


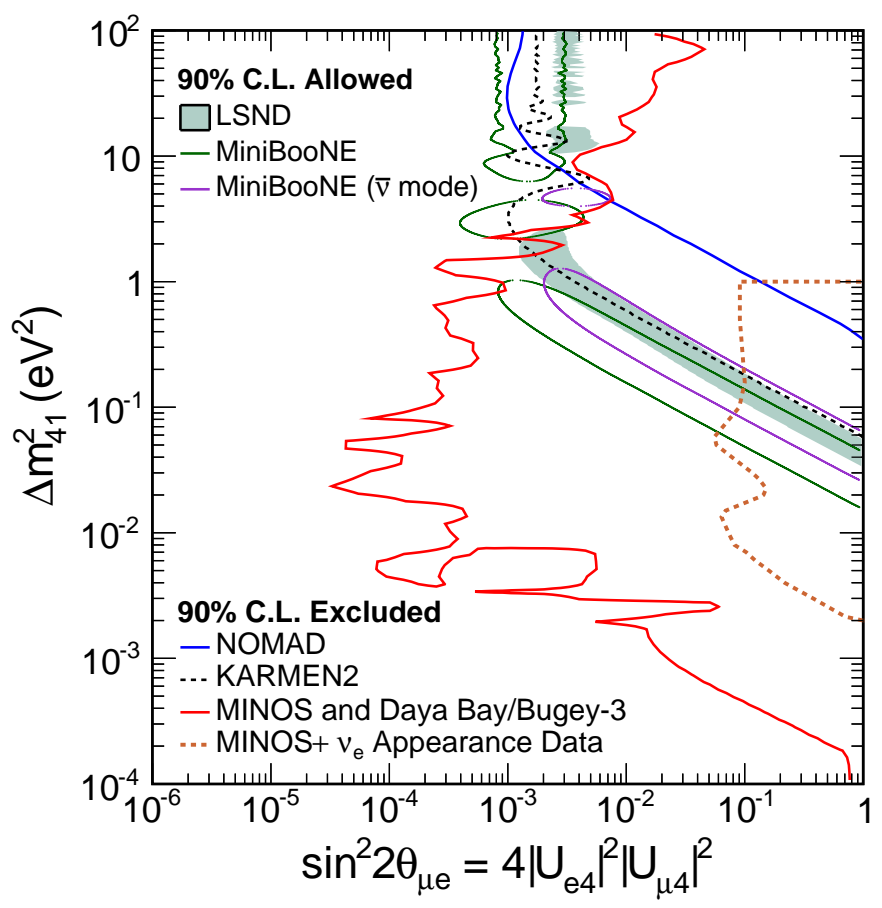

Figure 5: The 90\% exclusion limits from the MINOS and Daya Bay/Bugey-3 [17] combined analysis (red) and the MINOS $+v_{e}$ appearance result (dashed orange). These contours are compared to the LSND [18] and MiniBooNE [19] allowed regions, and the exclusion limits from KARMEN2 [20] and NOMAD [21].

\section{Sterile-driven $v_{e}$ Appearance}

The ME beam configuration used for MINOS+ provides an opportunity to directly probe the LSND/MiniBooNE phase-space by searching for anomalous appearance of electron neutrinos in the Far Detector. The analysis looks at the high energy part of the spectrum, above $6 \mathrm{GeV}$, away from the standard three-flavour oscillations and builds upon the framework developed for the MINOS three-flavour CC $v_{e}$ analyses [22]. A library-event-matching (LEM) algorithm is used to select the $\mathrm{CC} v_{e}$ candidate events from the large background of $\mathrm{NC}$ interactions, and a detailed description of this analysis is provided elsewhere in these proceedings. A total of 78 events were observed in the first year of MINOS+ data, compared to an expectation of 56.7, corresponding to a $2.3 \sigma$ excess. However, the $3+1$ sterile neutrino hypothesis does not provide a significantly improved description of this excess. The $90 \%$ C.L. exclusion contour obtained from the analysis is shown by the orange dashed line in Fig. 5, in agreement with the disappearance-based analysis.

\section{Conclusion}

The MINOS/MINOS+ experiment continues to perform high-precision measurements of neutrino oscillations. The updated three-flavour oscillation result with the inclusion of the first two years of MINOS+ data provides a very accurate measurement of the mass-splitting $\Delta m_{32}^{2}$. No evidence of a sterile neutrino was found across four analyses and strong limits spanning six orders of 
magnitude in $\Delta m_{41}^{2}$ on the existence of the sterile neutrino and sterile antineutrino were obtained. The addition of the final year of data (approximately half of the total MINOS+ exposure) will further improve the sensitivity of the measurements from the MINOS+ experiment over the next year.

\section{References}

[1] D. G. Michael et al. (MINOS Collaboration), Nucl. Instrum. Meth. A 596 190-228, 2008.

[2] P. Adamson et al. (MINOS Collaboration), Nucl. Instrum. Meth. A 806 279-306, 2016.

[3] S. Schael et al. (ALEPH Collaboration, DELPHI Collaboration, L3 Collaboration, OPAL Collaboration, SLD Collaboration, LEP Electroweak Working Group, SLD Electroweak Group, SLD Heavy Flavour Group), Phys. Rep. 427, 257, 2006.

[4] P. Adamson et al. (MINOS Collaboration), Phys. Rev. Lett. 112, 191801, 2014.

[5] P. Vahle, New results from NOvA, XXVII International Conference on Neutrino Physics and Astrophysics (Neutrino 2016), London, 2016.

[6] H. Tanaka, Status, recent results and plans for T2K, XXVII International Conference on Neutrino Physics and Astrophysics (Neutrino 2016), London, 2016.

[7] P. Adamson et al. (MINOS Collaboration), Phys. Rev. Lett. 117 151803, 2016.

[8] M. G. Aartsen et al. (IceCube Collaboration), Phys. Rev. Lett. 117 0712801, 2016.

[9] K. Abe et al. (Super Kamiokande Collaboration), Phys. Rev. D 91 052019, 2015.

[10] F. Dydak et al. (CDHSW Collaboration), Phys. Lett. B 134 281, 1984.

[11] I. E. Stockdale et al. (CCFR Collaboration), Phys. Rev. Lett. 52 1384, 1984.

[12] K. B. M. Mahn et al. (MiniBooNE and SciBooNE Collaborations), Phys. Rev. D 85 032007, 2012.

[13] I. E. Stockdale et al. (CCFR Collaboration), Z. Phys. C 27 53, 1985.

[14] G. Cheng et al. (MiniBooNE and SciBooNE Collaborations), Phys. Rev. D 86 052009, 2012.

[15] F. P. An et al. (Daya Bay Collaboration), Phys. Rev. Lett. 117 151802, 2016.

[16] Achkar B et al. (Bugey-3 Collaboration), Nucl. Phys. B 434 503, 1995.

[17] P. Adamson et al. (MINOS and Daya Bay Collaborations), Phys. Rev. Lett. 117 151801, 2016.

[18] A. Aguilar et al. (LSND Collaboration), Phys. Rev. D 64 112007, 2001.

[19] A. Aguilar-Arevalo et al. (MiniBooNE Collaboration), Phys. Rev. Lett. 110 161801, 2013.

[20] B. Armbruster et al. (KARMEN Collaboration), Phys. Rev. D 65 112001, 2002.

[21] P. Astier et al. (NOMAD Collaboration), Phys. Lett. B 570 19, 2003.

[22] P. Adamson et al. (MINOS Collaboration), Phys. Rev. Lett. 110 171801, 2013. 